\title{
Digital Kaleidoscope Patterns Design Based on Java Language
}

\author{
Ning Tang $^{1}$, Jianxia Su ${ }^{1, a}$, Li Zhao ${ }^{1}$ \\ ${ }^{1}$ Information school of Beijing Institute of Fashion Technology 100029 China \\ agxysujx@bift.edu.cn
}

Keywords: Kaleidoscope pattern; Java language; Algorithm Simulation

\begin{abstract}
In order to apply kaleidoscope patterns into textiles or garments, several methods are studied in this paper. Based on Java language, fractal algorithms, $M$ set algorithm and Julia set algorithm are used to simulate kaleidoscope patterns. The simulation results show that the kaleidoscope patterns programed can be used in textile and clothing. It provides users with more choice.
\end{abstract}

\section{Introduction}

In modern society, with the rapid development of science and technology, kaleidoscope pattern is widely used in clothing, textile and other decorative processing. A kaleidoscope is composed of three plane mirrors a simple prism, the main principle is each glass inside the kaleidoscope of light reflection effect. The kaleidoscope pattern is very beautiful and symmetry. It is necessary to simulate the kaleidoscope pattern with the help of computer.

\section{The methods designed to simulate the kaleidoscope pattern}

\section{Java Language tools to achieve a simple kaleidoscope pattern}

Based on the Java tools to draw kaleidoscope pattern. Graphical interface tool development of can be used for software programing ,The Graphics class is the base class for all graphics context, and the application can be performed on the component and the closed screen image. The object of the Graphics class is encapsulated to support Java programing. The following fractal algorithms are useful to the Graphics class, which is the basis of graphics. Java tools can achieve two times and three times, the linear curve of digital kaleidoscope patterns.

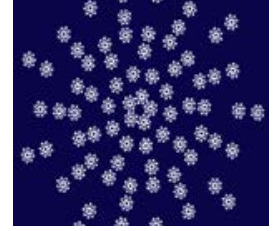

Fig. 1 small floral pattern

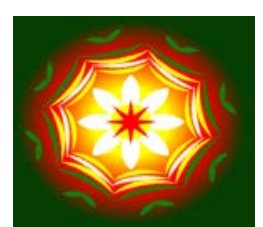

Fig. 2 two curves. pattern

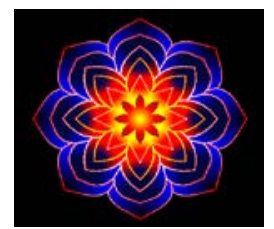

Fig. 3. three curves pattern

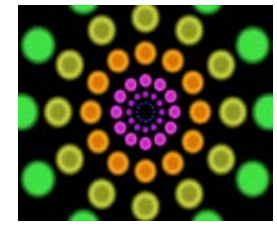

Fig. 4 rectangle fill gradient pattern

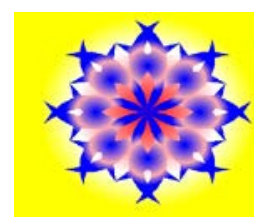

Fig. 5 three times curve blending pattern

Figure 1 is a pattern that is obtained by a flower pattern, copying and translating. While Figure 2 to figure 5 contains two and three times functions, The patterns can be based on the Radial Gradient Paint class in the Java class to provide a circular radiation and color gradient mode to simulate pattern.

Parameter implementation. In front of the kaleidoscope pattern, just by code generation of a pattern, if more variations are needed, Pattern parameters on the interface can be changed to achieve the controllability of the structure or color. So in construction pattern of the drawing window, the kaleidoscope of the input branches, color pattern, under the condition of random branching pattern number control is 
When "23" number is entered, the pattern is rotated 23 times, and the color value is obtained by default. As it is showed in Figure 6:

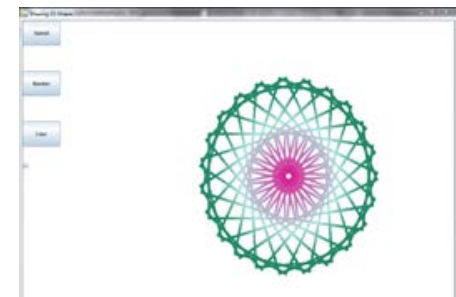

Fig. 6 Input parameters branch of the kaleidoscope pattern

\section{The digital kaleidoscope pattern Based on fractal algorithm}

Fractal can be regarded as a mathematical tool to describe nature. One of the core features of fractal is the fine structure, which can be seen in many times and the structure of the whole structure. There are many research results of fractal art in textile and clothing, Fractal algorithm can be divided into $\mathrm{M}$ set fractal, Julia set fractal and grammar composition and so on, the following are mainly $\mathrm{M}$ set fractal algorithm and Julia set fractal algorithm.

M algorithm kaleidoscope pattern. M set is a Mandelbrot Benoit in 1980. The expression of mathematical expression and Julia set is very similar, both of which are used in the two-dimensional iterative algorithm.. The Julia set is a complex structure which is realized on the $\mathrm{Z}$ plane by looking for the complex structure of the attraction domain and all the points on the boundary of the $\mathrm{Z}$, which is based on the $\mathrm{C}$ value, the first is to select the initial $\mathrm{Z}$ point, and then search the iteration matrix in the case of the $\mathrm{C}$ value of the transformation, and the structure of the matrix is recorded in the $\mathrm{C}$ plane of the parameter space.

The escape time algorithm is an important computational tool. There are two windows in the escape time algorithm, which is composed of the parameters of the $\mathrm{M}$ concentration parameter $\mathrm{C}$, the coordinates of the drawing window. $\mathrm{M}$ set drawing is achieved by all values of the parameters, so the size and location of the parameters of window is to determine the size of the M. M set scaling principle are according to the selected drawing window amplified region of parameter transformation for the range of parameters, in order to map out the $\mathrm{M}$ set local amplification kaleidoscope patterns.

$$
\begin{aligned}
& \Delta \mathrm{p}=\frac{p_{\text {max }}-p_{\text {min }}}{x_{\text {max }}-x_{\text {min }}} \quad \Delta \mathrm{q}=\frac{q_{\text {max }}-q_{\text {min }}}{y_{\text {max }}-y_{\text {min }}} \\
& \mathrm{p}=p_{\text {min }}+n_{p} * \Delta p\left(n_{p}=0,1,2, \cdots, n-1\right) \\
& \mathrm{q}=q_{\text {min }}+n_{q} * \Delta q\left(n_{q}=0,1,2, \cdots, m-1\right)
\end{aligned}
$$

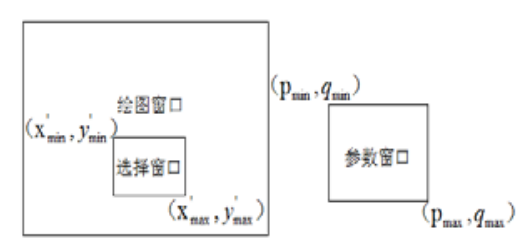

Fig. 7 Drawing, selection, parameter window of Mandelbrot

$$
\begin{aligned}
& \mathrm{p}=p_{\text {min }}+x^{*} \Delta p \\
& \mathrm{q}=q_{\text {min }}+y^{* \Delta} \mathbf{q}
\end{aligned}
$$

The above formula derivation can draw the coordinate parameters of zoom window area:

$$
\begin{aligned}
& \mathrm{p}_{\text {min }}^{\prime}=p_{\text {min }}+x_{\text {min }}^{\prime} * \Delta p \\
& \mathrm{q}_{\text {min }}^{\prime}=q_{\text {min }}+y_{\text {min }}^{\prime} * \Delta q \\
& \mathrm{P}_{\text {max }}^{\prime}=p_{\text {max }}+x_{\text {max }}^{\prime} * \Delta p \\
& \mathrm{q}_{\text {max }}^{\prime}=q_{\text {max }}+y_{\text {max }}^{\prime} * \Delta q
\end{aligned}
$$

At this point, the parameter value of the enlarged region is obtained, obtained and the above values can be drawn in the process of drawing the $M$ set, then we can draw the graphics of the 
selected area. And by adjusting the P, Q parameters, and the color When, in the two order $\mathrm{M}$ set drawing pattern selection, the kaleidoscope pattern is shown in Figure 8:
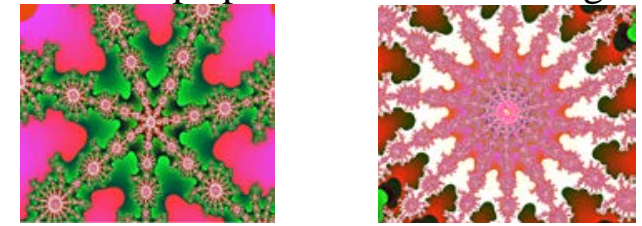

Fig.7 Mandelbrot set kaleidoscope pattern 1

Figure 7 is obtained by varying the magnification, the multiple is gradually increasing, the color is gradually changing, and the sense of the particles gradually becomes small. To the magnification of the limit, pattern distortion. For the Mandelbrot set, each site amplification of fractal graph is different, the difference between the two groups of pictures is rotation angle is different, the figure 8 is divergent rotation, and similar to Figure 98 in spiral shape and the distribution of color in the graphic is relatively smooth grainy not so obvious.
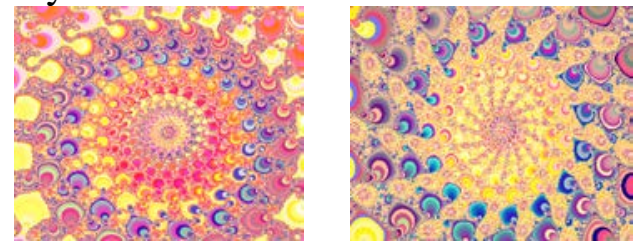

Fig.8Mandelbrot set kaleidoscope pattern 2

Julia algorithm kaleidoscope pattern. The Julia set is the same as the Mandelbrot set. It only needs to change the procedure of the part of the local amplification:

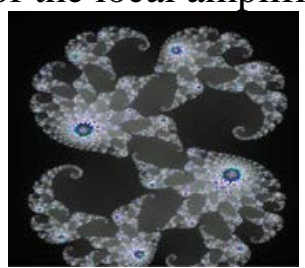

Fig. 9 Figure $\mathrm{p}=0.284 \mathrm{q}=0.011$ Julia set pattern

After amplification the kaleidoscope patterns as shown in Figure 11. From the following two images can be seen, the angle of the rotation of the pattern is the same, all the petals after the rotation of the iterative. And two amplitude magnification of figure is different, at the same time color in the process of rotation will continue to change.
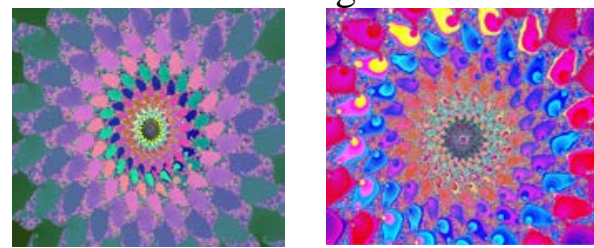

Fig. 10 Julia set $\mathrm{p}=0.284 \mathrm{q}=0.011$ amplification of kaleidoscope patterns

$\mathrm{P}$ values decreases will change the angle of rotation, Figure 12 is the $\mathrm{p}$ value obtained is 0.178 graphics. At this time of the rotation angle makes the pattern of presenting a kaleidoscope of linear divergence shape, and color of the algorithm and the graph 10 is same, can also be seen in the main tone of the two photos is same.
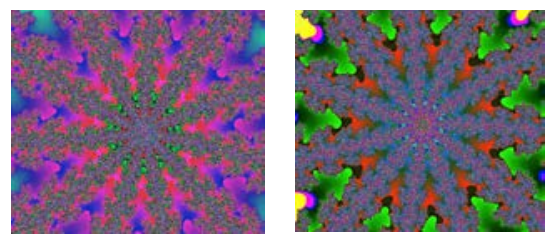

Fig. 11 Julia set $\mathrm{p}=0.178 \mathrm{q}=0.581$ amplification of kaleidoscope patterns 


\section{Summary}

Design of digital kaleidoscope patterns through the computer software provides tools, graphics, and image processing function and directly on a computer drawing pattern. Or by putting a programming method to obtain patterns, changing the parameters, through the modify color. The application of digital technology in the modem textile pattern design, create a new pattern style.

\section{Acknowledgments}

This paper is sponsored by KYJH02140201/025 of 2014 project of Beijing Science and Technology.

\section{Reference}

[1] Changhong Zhu, Based on the [D] technology of the semiconductor laser beam smoothing kaleidoscope. Hubei: Huazhong University of Science and Technology, 2012

[2] Xuhong Yang,RenChen Tang,Donggao Li, Fractal geometry, textile design and development and application of new tools [J]. Weaving technology. 2004:98-100

[3] Application of fractal patterns in fashion design [D]. Beijing: Beijing Institute of Fashion Technology, 2010

[4] White,Renee minus.Fractal fashion focuses on denim[N].THE NEW YORK AMSTER DAMNEWS 2002, Nov.28-Dec.4:17

[5] Yin Y, Yang F, Fan and S. Growth kinematics of fractal super snowflakes [J]. Chinese Science Bulletin 2010.7:573-580 